\title{
Effects of Endurance Exercise Training on Adipose Tissue Inflammatory Gene Expression in Elderly Rats with Diet-Induced Obesity
}

\author{
Kijin Kim* \\ ${ }^{1}$ Department of Physical Education, College of Physical Education, Keimyung University
}

Received: December 12, 2018
Accepted: January 9, 2018
Published online: January 31, 2019
Keywords:
Aging
Exercise
Inflammation
Macrophage polarization
Obesity

\section{ABSTRACT}

OBJECTIVES The study aimed to investigate the effects of the exercise training on inflammatory gene expression in adipose tissue via aging and obesity.

METHODS The study subjects were forty 10 -week old and 70 -week old male Wistar rats. The young and oldaged rats were divided into four groups of ten: control (non-exercise) group (Y-Non; O-Non) and exercise group (Y-Ex; O-Ex). After the exercise training, intergroup differences in body composition, oral glucose tolerance test result, and protein expression levels of IL-6, TNFa, IL-10, and IL-4 in adipose tissue, were compared.

RESULTS Both body weight and body fat-free mass were lower in old-aged rats than young rats; O-Non group, in particular, displayed the highest body fat mass and lowest fat-free mass. In oral glucose tolerance test result, old-aged rats exhibited higher blood glucose and insulin concentrations than young rats. TNF-a protein expression was higher in old-aged rats than young rats with the Y-Ex group displaying the lowest expression. IL-6 protein expression was significantly lower in the exercise group than in the control group for both old-aged and young rats $(\mathrm{p}<0.05)$. IL-10 protein expression was not significantly different between the exercise group and the control group for either old-aged or young rats. IL-4 protein expression was significantly lower in the exercise group of young rats $(p<0.05)$.

CONCLUSIONS Based on these findings, the analysis of the effects of exercise training on inflammatory gene expression in the adipose tissue of obese old-aged rats showed that the exercise training has effectively promoted the shift to $\mathrm{M} 2$ of cytokines secretion pattern related to obesity-induced pro-inflammation in old-aged rats and helped to improve insulin sensitivity.

(c) The Asian Society of Kinesiology and the Korean Academy of Kinesiology

\section{Introduction}

Aging is characterized by marked reduction in immune function with increasing cytokines such as CRP, IL-6, and TNF- a causing the progression of chronic inflammation $[1,2]$. Notably, inflammatory gene expression in adipose tissue increases in relation to the decrease in innate immune

*Correspondence: Kijin Kim, Department of Physical Education, College of Physical Education, Keimyung University, 1095 Dalgubeuldero, Dalseo-gu, Daegu, Korea Tel: +82-053-580-5256; Fax: +82-053-580-5314; E-mail: kjk744@kmu.ac.kr reactions, the main regulatory parameter of chronic inflammation [3]. Reduced physical activities and inadequate nutrient intake lead to substantially increased prevalence of metabolic diseases such as obesity and type II diabetes [4]. The adipose tissue of obese individual or animal contains an accumulation of macrophages that play a key role in immune function. Macrophages initially exist as monocytes, and while circulating in blood, they migrate to an area of bacterial infection or tissue damage where they differentiate 
into macrophages to destroy the source of infection or regenerate the damaged tissue [5]. The mechanism behind the macrophage influx into the adipose tissue and their phenotypes in each tissue has not yet been clearly elucidated. Recent studies reported two broad phenotypes of macrophages just as in lymphocytes: M1 phenotype is "classically activated" and cytokines such as IL-6, IL-1, IFN- $\gamma$, and TNF- $\alpha$ act as the mediator in LPS and Th1 for pro-inflammatory actions; M2 phenotype is "alternatively activated" with anti-proinflammatory properties and cytokines such as IL-4, IL-13, IL-10, TGF- $\beta$, and IL-1R ligands are expressed by Th2 [6]. Like LPS, the genes for endogenous ligands alongside saturated fatty acids and TLR4 complex undergo M1 polarization; i.e., the macrophage phenotype in obese individuals shifts from M2 to M1 of macrophage polarization, whereby the inflammatory actions in adipose tissue accelerates [7].

Obese or obesity-induced type II diabetes patients show significantly higher level of M1 markers and lower M2 markers than individuals with normal body weight [8]. The M1/M2 phenotype imbalance in peripheral blood monocytes is correlated with several metabolic parameters and arterial stiffness, and the cells in the liver and adipose tissues secrete anti-inflammatory cytokines (IL-4 or IL-3) for maintaining immune function homeostasis, and these in turn affect the macrophage phenotype in the respective tissues and lead to increased insulin resistance [9]. In patients with chronic diseases such as diabetes, insulin resistance increases prior to the pathogenesis, and although the exact mechanism inducing insulin resistance is unknown, PPAR delta among the relevant nuclear transcription factors has been speculated to play an important role in the shift to M2 phenotype and energy control in adipose tissue while increasing the fatty acid oxidation in myocytes or lipocytes [10]. However, such mechanisms remain unclear, and there is a lack of research linking them to the effective treatment method of exercise training. Recently, the positive changes in macrophage polarization induced by exercise training have been widely recognized, which brought growing emphasis on the positive effects of exercise and numerous studies are focusing on analyzing the relevant mechanisms [11]. Notably, Goh et al. [11] emphasized the need to analyze the different effects of exercise training from the resistance exercise including endurance exercise to the aerobic exercise where additional body weight tolerance has not been imparted. Nonetheless, the current level of investigation into inflammatory gene expression in adipose tissue in relation to $\mathrm{M} 1 / \mathrm{M} 2$ polarization and the changes in M1/M2 cytokines upon exercise training during the progression of aging is far from satisfactory.

The mechanism of inflammatory reaction during tissue damage, in particular, has not yet been clearly identified, and it is still unclear how the secretion of cytokines is correlated with the expression of immune function, based on the difficulties in early distinction between inflammatory necrosis and anti-inflammatory apoptosis. Continuing efforts to actively investigate the correlation of immune function to relevant inflammation in adipose tissue and the effects of exercise training as well as the increased prevalence of metabolic diseases during aging, are essential. Aging brings about the atrophy and dysfunction of skeletal and cardiac muscles, and potential causes for such phenomena have been analyzed in diverse ways. Nonetheless, the recently recognized correlation between mitochondrial dysfunction and abnormal immune function has not yet been given adequate attention, and there is more serious lack of studies on inflammatory gene expression in adipose tissue. To study the changes in correlation between mitochondrial biogenesis and immune function as well as the inflammatory gene expression in adipose tissue upon obesity in aging, is anticipated to be a work of considerable value as it will lay the essential foundation for applied physiology-based analysis. Furthermore, by clearly defining the effects on aginginduced inflammatory gene expression in adipose tissue, of a long-term aerobic exercise training, the study will considerably assist the prevention and treatment of aging-related diseases and suggest appropriate solutions in response to population aging.

This study thus verified the importance of exercise training in the prevention of aging by comparatively analyzing young and old-aged rats undergoing high fat diet and subsequent 12week endurance exercise training, then analyzing the effects of the exercise training on the inflammatory gene expression in adipose tissue caused by obesity in aging. 


\section{Methods}

This study aims to investigate the effects of exercise training on the expression of cytokines related to macrophage polarization in the adipose tissue of obese young and old-aged rats.

\section{Subjects}

The study subjects were twenty 10 -week and twenty 70 -week male Wistar rats (forty rats in total). For animal breeding, after a week of pre-breeding for the rats to adapt to the environment, a 12-week treatment was given to each group, and during pre-breeding, all rats were kept in a cage with just one other rat. The daily light-dark cycle involved the mice adapting to $12 \mathrm{~h}$ of lightness and $12 \mathrm{~h}$ of darkness, and the breeding room temperature was kept at $24 \pm 1{ }^{\circ} \mathrm{C}$ and relative humidity within $60 \%$, while the rats were allowed to drink and feed freely.

For grouping, the rats were randomly divided based on young rats, old-aged rats, and exercise training (Ex) into four groups of ten: the control (non-exercise training) group of young rats (Y-Non) and the exercise group (Y-Ex) and the control group of old-aged rats (O-Non) and exercise group (O-Ex).

\section{Experimental design}

\section{Diet for laboratory animals}

High-fat diet comprised carbohydrate $30 \%$, fat $50 \%$, and protein $20 \%$ of total calorie, while the general diet comprised carbohydrate $64.5 \%$, fat $11.8 \%$, and protein 23.5 $\%$ of total calorie, then they were mixed with vitamins ( $22 \mathrm{~g} /$ kg Teklad vitamins mix no. 40077), minerals (51 g/kg Teklad mineral mix no. 170915), methionine (5 g/kg, Teklad Premier no.10850), and choline chloride (1.3 g/kg) [12]. The rats were allowed to drink water and feed freely.

\section{Exercise training program}

The rats were made to perform endurance exercise of treadmill running. The exercise intensity for the rat was $\mathrm{VO}_{2} \max 60-75 \%$ as it was carried out five times a week for the period of 12 weeks. The rats were given a week to adapt to the breeding room, then at week one of exercise training, they were made to perform a warm-up exercise for $5 \mathrm{~min}$ at the speed of $8 \mathrm{~m} / \mathrm{min}$, then for $10 \mathrm{~min}$ (twice a day) at the speed of $18 \mathrm{~m} / \mathrm{min}$. At weeks $2-3$, while the speed was maintained at the same $18 \mathrm{~m} / \mathrm{min}$ as week one, the rats were made to exercise for $30 \mathrm{~min}$ (twice a day) at week 2 , then for $40 \mathrm{~min}$ (twice a day) at week 3. At weeks $4-6$, the exercise intensity was increased to $5 \mathrm{~min}$ of warm-up at $10 \mathrm{~m} / \mathrm{min}$ followed by $60 \mathrm{~min}$ of exercise at $22 \mathrm{~m} / \mathrm{min}$. At weeks $7-8$, the intensity was $5 \mathrm{~min}$ of warm-up at $15 \mathrm{~m} / \mathrm{min}$ followed by $60 \mathrm{~min}$ of exercise at $22 \mathrm{~m} / \mathrm{min}$. For the animal exercise training employed in this study, the methods of previous studies $[13,14]$ were modified for complementation and used.

\section{Measurement items and methods}

Body composition The animals prior to sacrifice were given intraperitoneal injection of $70 \mathrm{mg} / \mathrm{kg}$ of ketamine (JW Pharmaceutical, Korea) and $10 \mathrm{mg} / \mathrm{kg}$ of Xylazine (JW Pharmaceutical, Korea) for general anesthesia. The body fat mass and fat-free mass were measured using DEXA (DSC-3000, Aloka, Tokyo, Japan) which has a small animal total body option.

Oral glucose tolerance test After the 12-week treatment, the animals were made to rest for $48 \mathrm{~h}$ to remove the last bout exercise effect, followed by $12 \mathrm{~h}$ fasting. Blood at rest was collected from the tail of the animal, and after oral administration of $1 \mathrm{~g} / \mathrm{kg}$ of $50 \%$ glucose solution, $400 \mu \mathrm{l}$ of blood was collected at 15, 30, 60, and $120 \mathrm{~min}$. The collected blood was treated with an anticoagulant (50 $\mu$ l heparin) and centrifuged ( $1500 \mathrm{~g}, 15 \mathrm{~min}$ ) for isolating the plasma, which was stored at $-80{ }^{\circ} \mathrm{C}$ until the subsequent analysis. The animals whose blood has been extracted were intravenously injected with $2.5 \mathrm{ml}$ of $0.9 \%$ saline solution to complement the lost blood. After three days of recovery, the animals were anesthetized for tissue extraction [15].

Tissue extraction and blood collection After the oral glucose tolerance test, followed by three days of recovery and $12 \mathrm{~h}$ fasting, the animals were anesthetized using pentobarbital sodium (5 mg per $100 \mathrm{~g}$ of body mass) for blood collection, after which epididymal fat pads were extracted and weighed. The extracted tissues were clamp frozen and stored at $-80{ }^{\circ} \mathrm{C}$ until the subsequent analysis. After the tissue extraction, $5 \mathrm{ml}$ of blood from 
abdominal artery was collected and treated with an anticoagulant (50 $\mu \mathrm{l}$ heparin). The collected blood was centrifuged (1500 g, $15 \mathrm{~min}$ ) for isolating the plasma, which was stored at $-80{ }^{\circ} \mathrm{C}$ until the subsequent analysis.

Analysis of blood variables For measuring blood glucose level, an automated blood glucose analyzer (YSI 2300, Springfield, USA) was used, and for measuring insulin, a rat insulin ELISA kit (Mercodia, Uppsala, Sweden) was used, and for measuring TNF- $\alpha$, IL-4, IL-6, and IL-10, rat ELISA kits (Biosource International Inc., USA) was used.

Western blotting The tissues were homogenized using ice-cold buffer [250 $\mathrm{mM}$ sucrose, $10 \mathrm{mM}$ HEPES/1 mM EDTA(pH 7.4), 1 mM Pefabloc(Roche), 1 $\mathrm{mM}$ EDTA, $1 \mathrm{mM} \mathrm{NaF}, 1 \mathrm{~g} / \mathrm{ml}$ aprotinin, $1 \mathrm{~g} / \mathrm{ml}$ leupeptin, $1 \mathrm{~g} / \mathrm{ml}$ pepstatin, $0.1 \mathrm{mM} \mathrm{bpV}$ (phen), $2 \mathrm{mg} / \mathrm{ml}$ glycerophosphate] [16]. The homogenized samples were given a freeze-thaw process three times, then centrifuged (700 g, $10 \mathrm{~min}$ ). Following the method of Lowry et al.[17], protein quantification was carried out. The samples were dissolved in Laemmli buffer and aliquoted to SDS-polyacrylamide gel for electrophoresis. For antibody-based immunoblotting, the following antibodies were used: IL-6, TNF- $a$, IL-10, and IL-4. After the use of secondary antibodies for each, ECL was used for visualization and densitometry (sigma-plot 8.0 system) was used for quantification.

\section{Statistical analysis}

All measured data were analyzed using SPSS-PC (Version 21.0) for calculating the mean and standard deviation for each group. The intergroup differences in terms of dependent variables, one-way analysis of variance (ANOVA) was used, and for post-hoc, Duncan's method was used. The significance level was set as $\mathrm{p}<0.05$.

\section{Results}

The result of analyzing the body composition indices used in this study is given in Figure 1. As shown, both the body weight and fat-free mass were lower in old-aged rat groups than in young rat groups; in particular, the O-Non showed the highest fat mass and the lowest fat-free mass. Thus, it was thought that the exercise training program was effective for body fat reduction in old-aged rats.

The result of oral glucose tolerance test is given in Figure 2, where the blood glucose and insulin levels were higher in old-aged rat groups than in young rat groups. Thus, the lack of exercise and aging were thought to have negative influence on insulin sensitivity.

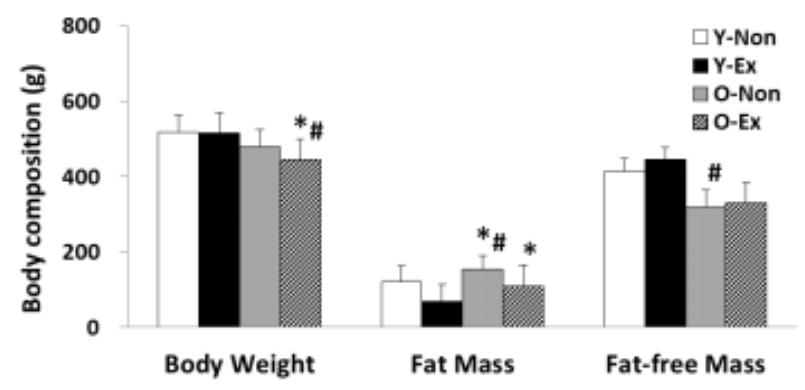

Figure 1. Results of body composition (* $p<0.05$ Compared to $Y$-Non group)
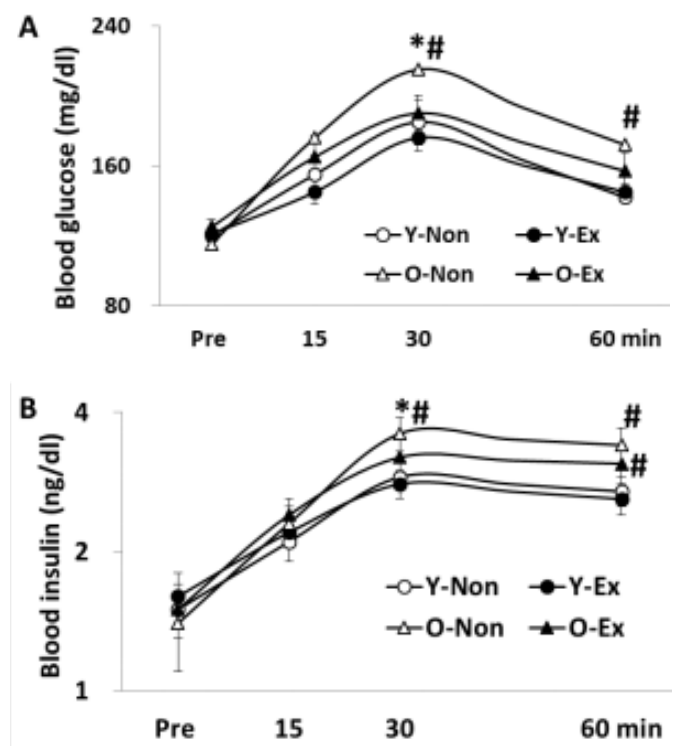

Figure 2. Changes of glucose $(A)$ and insulin (B) in OGTT ( ${ }^{*} p<0.05$ Compared to $Y$-Non group)

NF- $\alpha$ and IL- 6 protein expression in adipose tissue are as shown in Figure 3. TNF-a protein expression was higher in old-aged rat groups than young rat groups; in particular, the YEx showed the lowest TNF- $\alpha$ protein expression. IL-6 protein expression was significantly lower $(\mathrm{p}<0.05)$ in the exercise group than the control for both old-aged and young rats. Thus, the lack of exercise and aging were thought to increase TNF$a$ and IL- 6 protein expression in adipose tissue. 
IL-10 and IL-4 protein expression in adipose tissue are as shown in Figure 4. IL-10 protein expression did not exhibit significant differences between the exercise group and the control for both old-aged and young rats whereas IL-4 protein expression was significantly lower $(\mathrm{p}<0.05)$ in the Y-Ex. Thus, the lack of exercise and aging were thought to have a potential to increase IL- 4 protein expression in adipose tissue while having insignificant influence on IL-10 protein expression.

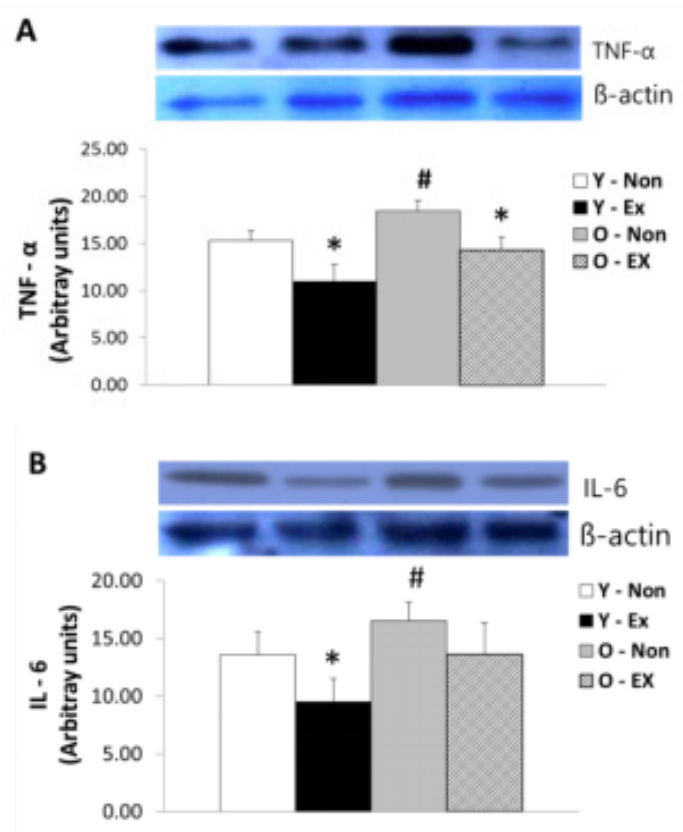

Figure 3. Results of TNF- $\alpha$ (A) and IL-6 (B) protein expressions in adipose tissue ( $*$ p $<0.05$ Compared to Non-exercise group, \# $p<0.05$ Compared to Young group)

The plasma TNF- $\alpha$ and IL- 6 concentrations are as shown in Figure 5. TNF- $\alpha$ concentration in blood was higher in old-aged rat groups than young rat groups; in particular, the Y-Ex showed the lowest plasma TNF- $a$ concentration. IL-6 concentration in blood was significantly lower $(\mathrm{p}<0.05)$ in the exercise group than the control for both old-aged and young rats. Thus, the lack of exercise and aging were thought to have increased plasma TNF- $\alpha$ and IL- 6 concentrations upon obesity.

The plasma IL-10 and IL-4 concentrations are as shown in Figure 6. IL-10 and IL-4 concentrations in blood did not exhibit significant differences between the exercise group and the control for both old-aged and young rats; nevertheless, the exercise groups had a trend of higher concentrations. Thus, the lack of exercise and aging were thought to have a potential to decrease plasma IL-10 and IL-4 concentrations but without exerting a significant influence.

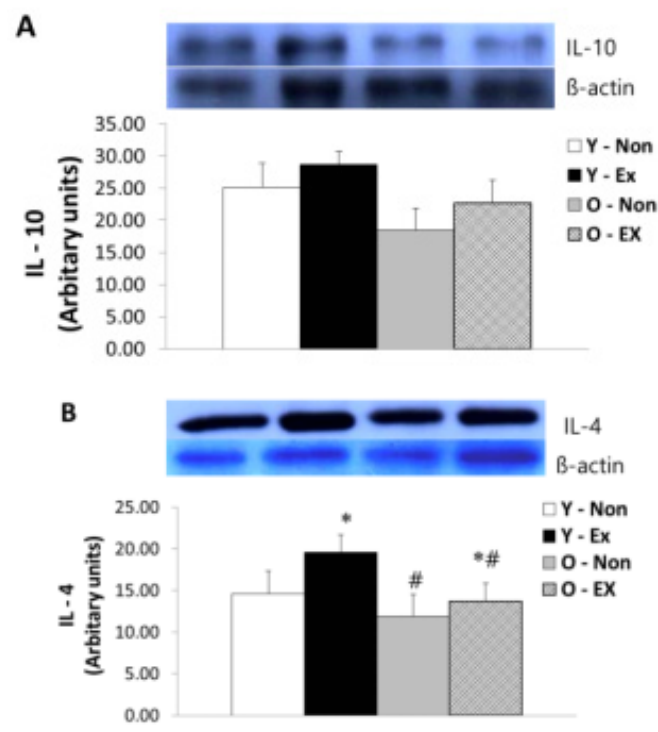

Figure 4. Results of IL-10 (A) and IL-4 (B) protein expressions in adipose tissue ( ${ }^{*}<<0.05$ Compared to Non-exercise group, \# $p<0.05$ Compared to Young group)

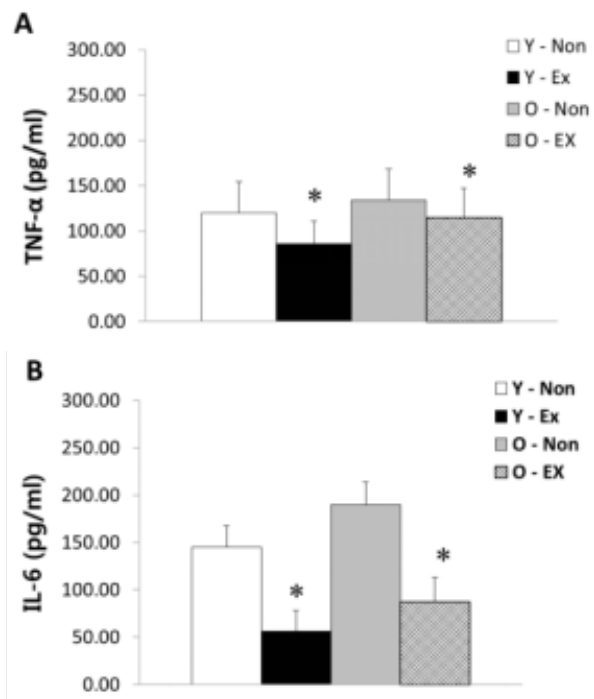

Figure 5. Results of TNF- $a(A)$ and IL-6 (B) blood concentrations $\left({ }^{*} \mathrm{p}<0.05\right.$ Compared to Non-exercise group) 


\section{A}

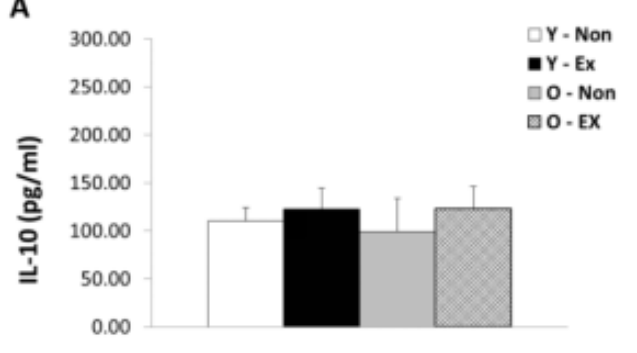

B

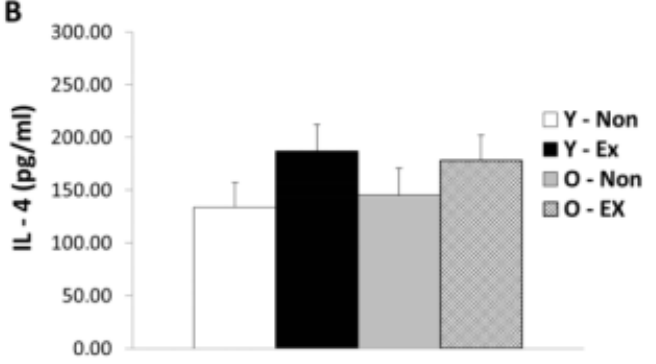

Figure 6. Results of II-10 (A) and IL-4 (B) blood concentrations

\section{Discussion}

This study analyzed the inflammatory gene expression in adipose tissue and the aggravation of insulin resistance that occur during aging in old-age and diet-induced obesity progression. The findings are expected to be particularly useful as essential data elucidating the mechanism behind immune function in the progression of obesity and aging, especially the increase in adipose tissue, sarcopenia of skeletal muscle, and macrophage polarization in adipose tissue. By analyzing the efficacy of exercise training on preventing and delaying such changes, the study is likely to provide clearer evidence.

Obesity has been regarded as a grave risk factor for type II diabetes, hyperlipidemia, cardiovascular disease, and cancer, as it induces insulin resistance $[18,19]$. Although pro-inflammation is considered the key factor in the mechanism of obesity-induced insulin resistance [20,21], the detailed pathways remain unclear. During obesity-induced inflammation, macrophage polarization plays a pivotal role, and TNF- $\alpha$ [22] and IL-10 [6] are thought to be prominent M1 cytokine involved in pro-inflammation and M2 cytokine involved in anti-pro-inflammation, respectively. Thus, the results of this study analyzing the cytokines in adipose tissue when obesity is induced and exercise training is performed are expected to provide crucial evidence for understanding macrophage polarization.

Reduced immune function has been suggested as a factor related to the mechanism of obesity-induced insulin resistance [23]; however, insulin resistance did not improve when immune function was activated in diet-induced obese mice [24] and distinguished induction of insulin resistance upon pro-inflammation could not be found [25], implying unclear evidence. But the findings in this study showed a marked difference in insulin resistance between young rats and old-aged rats, both groups displaying aggravation of insulin resistance during diet-induced obesity. Thus, increase in adipose tissue due to aging and obesity was clearly verified as a key factor promoting insulin resistance [26,27]. Also, exercise training was found to have improved insulin resistance in both groups of rats, suggesting aerobic exercise may be an effective treatment for insulin resistance. Especially, exercise training during aging process, in particular, displayed a significant response the prevention of body fat mass accumulation.

Aging and obesity influence the increased prevalence of metabolic diseases such as diabetes as well as insulin resistance by having a crucial impact on macrophage polarization related to pro-inflammation in adipose tissue [28]. In this light, studies have analyzed diverse factors involved in the development of macrophage polarization in various tissues, highlighting $\mathrm{C} /$ EBP $\alpha$, PPAR $\gamma$ and PPAR $\delta$ as the relevant factors $[10,29,30]$. This study set out to provide clearer evidence by analyzing the protein expression of cytokines involved in the polarization of macrophages secreted in adipose tissue, the fundamental pro-inflammatory tissue. The phenomenon of macrophage polarization due to aging and obesity has been investigated by various previous studies that frequently reported the increased probability of pro-inflammation caused by relative activation of M1 lineage [31,32]. This study has also found increased concentrations of IL- 6 and TNF- $\alpha$ in blood and protein expression of IL- 6 and TNF- $\alpha$ in adipose tissue in both groups of diet-induced obese young and old-aged rats, which clearly confirmed the shift to M1 of macrophage polarization. It has been widely emphasized that macrophage polarization underlies increased secretion of IL- 6 and TNF- $\alpha$, the M1 lineage cytokines that are extensively activated in obese state 
to cause insulin resistance [33], and active release of IL-10 and IL-4 when obesity and pro-inflammation are relatively ameliorated [6].

Recently, the importance of regular exercise in the treatment of obesity and prevention of aging has been widely proposed by diverse studies [34]. For this, emphasis has been placed on the provision of more definite evidence, while it is clear that increased energy consumption through exercise training program has indisputable effects on body fat reduction and treatment of aging-related factors. In time, evidence was found to suggest that exercise training program is effective for controlling the factors that influence pro-inflammation in tissues of the body [11]. In line with this and to clearly verify the suggestion, this study investigated diet-induced obese young rats and old-aged rats after a 12-week aerobic exercise. Analyzing the pattern of cytokine secretion related to macrophage polarization showed significant decrease in blood concentrations of IL- 6 and TNF- $\alpha$ and their protein expression in adipose tissue accompanied by marked activation of IL-4 and IL-10 protein expression in adipose tissue. This suggested that the aerobic exercise positively transform the pattern of cytokine secretion related to macrophage polarization in adipose tissue and effectively prevent pro-inflammation. Such findings have gained probability with the reports that exercise training program in obesity induction ameliorates toll-like receptor 4 signaling pathway involved in pro-inflammation [35], activates anti-pro-inflammatory factors [36], and reduces the secretion of CD68 and TNF- $\alpha$ in white adipose tissue [37]. The significant shift to $M 2$ of macrophage polarization reported by Oliveira et al. [38], where TNF- $\alpha$ and IL-1 MCP1 mRNA expression was analyzed in white adipose tissue of diet-induced obese rats after a long period of swimming exercise, lends further support to the findings of this study. $\operatorname{PPAR} \gamma$ has recently been proposed as a critical factor uniting exercise training and the significant shift to M2 of macrophage polarization [39] although further research should continue the efforts to analyze the detailed mechanism and parameters.

As the findings indicate, to use molecular biological perspectives to confirm the role of adipose tissue in proinflammation, a major cause of reduced skeletal muscle function due to immobilization as well as metabolic diseases in obesity induction and aging, and to investigate the patterns of molecular biological changes to verify the effects of exercise training on the rehabilitation of immobilized skeletal muscle after fall accidents, are likely to provide critical evidence for more definite design of exercise training program for preventing aging and for rehabilitation treatment. In particular, comparing the different progression in young and old-aged rats in light of such molecular biological studies, indispensable evidence may be produced for specifying the characteristics of the rehabilitation program for aging rats.

\section{Conclusion}

In conclusion, this study investigated the effects of exercise training on inflammatory gene expression in the adipose tissue of obese old-aged rats, and the results showed that the aerobic exercise is effective for promoting the shift to M2 of cytokine secretion pattern related to obesity-induced pro-inflammation in old-aged rats and for improving insulin sensitivity (Figure 7).

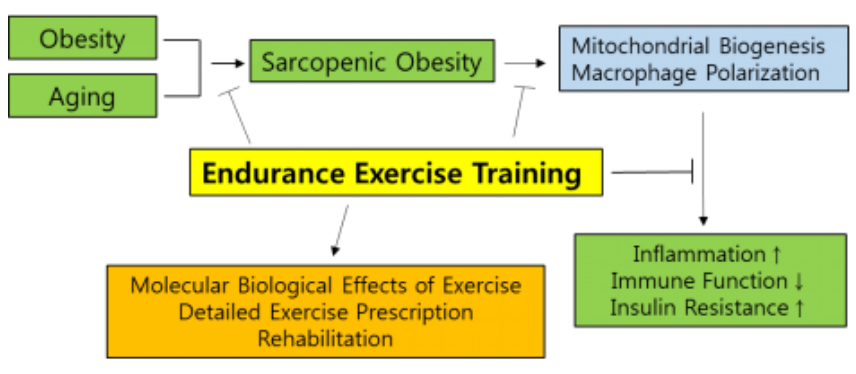

Figure 7. Summary of application of this study's results

\section{Acknowledgments}

This research was supported by the Keimyung University Research Grant of 2017.

\section{Conflicts of Interest}

The author declare no conflicts of interest.

\section{References}

1. Franceschi C, Bonafe M, Valensin S. Human immunosenescence: the prevailing of innate immunity, 
the failing of clonotypic immunity, and the filling of immunological space. Vaccine, 2000; 18:1717-1720.

2. Franceschi $\mathrm{C}$, Bonafe $\mathrm{M}$, Valensin $\mathrm{S}$ et al., Inflamm-aging. An evolutionary perspective on immunosenescence. Ann N Y Acad Sci. 2000; 908:244-254.

3. Suganami T, Ogawa Y. Adipose tissue macrophages: their role in adipose tissue remodeling. J Leukoc Biol. 2010; 88:33-39.

4. WHO. Obesity and Overweight. Fact Sheet. 2016; Available online at: http://www.euro.who.int/en/health-topics/ noncommunicable-diseases/obesity/obesity

5. Rocha VZ, Libby P. Obesity, inflammation, and atherosclerosis. Nat Rev Cardiol. 2009; 6:399-409.

6. Lumeng CN, Bodzin JL, Saltiel AR. Obesity induces a phenotypic switch in adipose tissue macrophage polarization. J Clin Invest. 2007; 117:175-184.

7. Catrysse L, van Loo G. Adipose tissue macrophages and their polarization in health and obesity. Cellular Immunology, 2018; https://doi.org/10.1016/j. cellimm.2018.03.001

8. Satoh N, Shimatsu A, Himeno A et al., Unbalanced M1/ M2 phenotype of peripheral blood monocytes in obese diabetic patients: effect of pioglitazone. Diabetes Care. 2010; 33(1):e7. doi: 10.2337/dc09-1315.

9. Bouhlel MA, Derudas B, Rigamonti E et al., PPARactivation primes human monocytes into alternative M2 macrophages with anti-inflammatory properties. Cell Metab. 2007; 6:137-143.

10. Odegaard JI, Ricardo-Gonzalez RR, Red Eagle A et al., Alternative M2 activation of Kupffer cells by PPARdelta ameliorates obesity-induced insulin resistance. Cell Metab. 2008; 7:496-507.

11. Goh J, Goh KP, Abbasi A. Exercise and adipose tissue macrophages: new frontiers in obesity research? Front Endocrinol. 2016; 7:65. doi: 10.3389/fendo.2016.00065

12. Hancock CR, Han DH, Chen M et al., High-fat diets cause insulin resistance despite an increase in muscle mitochondria. Proc Natl Acad Sci. 2008; 105(22):78157820.

13. De Angelis K, Wichi RB, Jesus WR et al., Exercise training changes autonomic cardiovascular balance in mice. J
Appl Physiol. 2004; 96(6):2174-2178.

14. Holloszy JO. Biochemical adaptations in muscle. J Biol Chem. 1967; 242(9):2278-2282.

15. Henriksen EJ, Teachey MK, Lindborg KA, Diehl CJ, Beneze AN. The high-fat-fed lean Zucker rat: a spontaneous isocaloric model of fat-induced insulin resistance associated with muscle GSK-3 overactivity. Am J Physiol Regul Integr Comp Physiol. 2008; 294(6):R1813-1821.

16. Margolis B, Ziberstein A, Franks C et al., Effect of phospholipase C-gamma overexpression on PDGFinduced second messengers and mitogenesis. Science, 1990; 248(4955):607-610.

17. Lowry EC, Blumber JM, Rhea RL, Ranson JP. Serum levels of orally administered penicillin. U S Armed Forces Med J. 1951; 2(2):265-270.

18. Odegaard JI, Chawla A. Pleiotropic actions of insulin resistance and inflammation in metabolic homeostasis. Science, 2013; 339(6116):172-177.

19. Park J, Morley TS, Kim M, Clegg DJ, Scherer PE. Obesity and cancer-mechanisms underlying tumour progression and recurrence. Nat Rev Endocrinol. 2014; 10(8):455465.

20. Lackey DE, Olefsky JM. Regulation of metabolism by the innate immune system. Nat Rev Endocrinol. 2016; 12(1):15-28.

21. McLaughlin T, Ackerman SE, Shen L, Engleman E. Role of innate and adaptive immunity in obesity-associated metabolic disease. J Clin Invest. 2017; 127(1):5-13

22. Weisberg SP, Weisberg SP, McCann D et al., Obesity is associated with macrophage accumulation in adipose tissue. J Clin Invest. 2003; 112(12):1796-1808.

23. Rosen ED, Spiegelman BM. What we talk about when we talk about fat. Cell. 2014; 156(1-2):20-44.

24. Lee YS, Li P, Huh JY et al., Inflammation is necessary for longterm but not short-term high-fat diet-induced insulin resistance. Diabetes, 2011; 60(10):2474-2483.

25. Tian J, Dang HN, Yong J et al., Oral treatment with $\gamma$-aminobutyric acid improves glucose tolerance and insulin sensitivity by inhibiting inflammation in high fat diet-fed mice. PloS ONE, 2011; 6(9) e25338.

26. Mau T, Yung R. Adipose tissue inflammation in aging. 
Experimental Gerontology, 2018; 105:27-31.

27. Shimobayashi M, Albert V, Woelnerhanssen B et al., Insulin resistance causes inflammation in adipose tissue. Clin Invest. 2018; 128(4):1538-1550. https://doi. org/10.1172/JCI96139.

28. Olefsky JM, Glass CK. Macrophages, inflammation, and insulin resistance. Annu Rev Physiol. 2010; 72:219-246.

29. Lee B, Qiao L, Lu M et al., C/EBPa regulates macrophage activation and systemic metabolism. Am J Physiol Endocrinol Metab. 2014; 306:E1144-E1154.

30. Odegaard JI, Ricardo-Gonzalez RR, Goforth MH et al., Macrophage-specific PPARgamma controls alternative activation and improves insulin resistance. Nature, 2007; 447:1116-1120.

31. Lumeng CN, DelProposto JB, Westcott DJ, Saltiel AR. Phenotypic switching of adipose tissue macrophages with obesity is generated by spatiotemporal differences in macrophage subtypes. Diabetes, 2008; 57:3239-3246.

32. Weisberg SP, Hunter D, Huber R et al., CCR2 modulates inflammatory and metabolic effects of high-fat feeding. J Clin Invest. 2006; 116:115-124.

33. Dandona P, Weinstock R, Thusu K, Abdel-Rahman E, Aljada A, Wadden T. Tumor necrosis factor-alpha in sera of obese patients: fall with weight loss. J Clin Endocrinol Metab. 1998; 83:2907-2910

34. Mason AE, Hecht FM, Daubenmier JJ et al., Weight loss maintenance and cellular aging in the supporting health through nutrition and exercise study. Psychosomatic Medicine. 2018; 80(7):609-619, DOI: 10.1097/ PSY.0000000000000616

35. Oliveira AG, Carvalho BM, Tobar N et al., Physical exercise reduces circulating lipopolysaccharide and TLR4 activation and improves insulin signaling in tissues of DIO rats. Diabetes, 2011; 60:784-796.

36. Xu X, Ying Z, Cai M et al., Exercise ameliorates high-fat diet-induced metabolic and vascular dysfunction, and increases adipocyte progenitor cell population in brown adipose tissue. Am J Physiol. 2011; 300:R1115-R1125.

37. Bruun JM, Helge JW, Richelsen B, Stallknecht B. Diet and exercise reduce lowgrade inflammation and macrophage infiltration in adipose tissue but not in skeletal muscle in severely obese subjects. Am J Physiol Endocrinol Metab. 2006; 290:E961-E967.

38. Oliveira AG, Araujo TG, Carvalho BM et al., Acute Exercise Induces a Phenotypic Switch in Adipose Tissue Macrophage Polarization in Diet-Induced Obese Rats. Obesity, 2013; 21:2545-2556. doi:10.1002/oby.20402.

39. Silveira LS, Batatinha HAP, Castoldi A et al., Exercise rescues the immune response fine-tuned impaired by peroxisome proliferator-activated receptors $\gamma$ deletion in macrophages. J Cell Physiol. 2019; 234(4):5241-5251 